\title{
RINGS OF CONTINUOUS FUNCTIONS ON OPEN CONVEX SUBSETS OF $R^{n}$
}

\author{
LYLE E. PURSELL ${ }^{1}$
}

0. Introduction. If $h$ is a homeomorphism from a topological space $X$ onto another space $Y$, then one can easily show that the correspondence $f \rightarrow f \circ h^{-1}$ is an isomorphism from $C(X)$, the ring of all real continuous functions on $X$, on to $C(Y)$, the ring of all real continuous functions on $Y$. The converse problem-given an isomorphism $\phi$ from $C(X)$ onto $C(Y)$ to show that there exists a homeomorphism $h$ from $X$ onto $Y$ such that $\phi f=f \circ h^{-1}$-is more difficult and is one of the problems which has motivated much of the research on rings of continuous functions. The isomorphism $\phi$ is usually constructed by using fixed, maximal ideals. An ideal $M$ in $C(X)$ is fixed if there exists a point $x_{0}$ of $X$ such that $f\left(x_{0}\right)=0$ for all $f$ in $M$. If $X$ is completely regular, then the set $M(x)$ of all functions in $C(X)$ which are zero at $x$ is a fixed, maximal ideal and all fixed, maximal ideals are of this form. If one can show that the property of a maximal ideal being fixed is invariant under ring isomorphisms and if $X$ and $Y$ are completely regular, then one can show that the correspondence

$$
x \rightarrow M(x) \rightarrow \phi(M(x))=M(y) \rightarrow y,
$$

where $M(y)$ is the set of all functions in $C(Y)$ which are zero at a point $y$ of $Y$, is the desired homeomorphism $h$. If $X$ and $Y$ are compact, then all maximal ideals are fixed, but if $X$ and $Y$ are not compact, then there are maximal ideals in $C(X)$ and $C(Y)$ which are not fixed. (Nonfixed ideals are called "free" ideals.) The maximal, fixed ideals in $C(X)$ have been algebraically characterized for certain classes of noncompact spaces, $X$, including the class of all separable metric spaces and the class of all normal Hausdorff spaces whose points are $G$-delta sets, but these characterizations are complicated and/or difficult to establish. (References: Gelfand and Kolmogoroff [1], Hewitt [4], Pursell [5], and Gillman and Jerison [2].)

In this paper we give a construction of the homeomorphism $h$ for the case $X$ and $Y$ are open convex subsets of $R^{n}$ which does not depend on an algebraic characterization of fixed, maximal ideals. This method of construction will also give us the theorem:

Presented to the Society, October 5, 1967; received by the editors February 14, 1967.

1 The author is indebted to the referee for correcting several errors in the original manuscript. 
If $C^{\infty}(X)$ and $C^{\infty}(Y)$ are the rings of all real, infinitely differentiable functions on the open convex subsets $X$ and $Y$ of $R^{n}$, respectively and $\phi$ is an isomorphism from $C^{\infty}(X)$ onto $C^{\infty}(Y)$, then there is a diffeomorphism $h$ from $X$ onto $Y$ such that $\phi f=f \circ h^{-1}$ for all $f$ in $C^{\infty}(X)$.

Our construction depends upon two properties of infinitely differentiable functions on open convex subsets $X$ of $R^{n}$ :

(0.1) A function in $C(X)$ can be uniformly approximated on $X$ by a function in $C^{\infty}(X)$.

(0.2) If $f$ is in $C^{\infty}(X)$, then to each point $\bar{a}=\left(a_{1}, a_{2}, \cdots, a_{n}\right)$ in $X$, there corresponds an $n$-tuple $\left(f_{\bar{a}, 1}, f_{\bar{a}, 2}, \cdots, f_{\bar{a}, n}\right)$ of functions in $C^{\infty}(X)$ such that

$$
f(\bar{r})=f(\bar{a})+\sum_{i=1}^{n}\left(r_{i}-a_{i}\right) f_{\bar{a}, i}(\bar{r})
$$

for all $\bar{r}=\left(r_{1}, r_{2}, \cdots, r_{n}\right)$ in $X$.

The proof of the first of these properties is given in detail in [6] for $X=R$ and is outlined there for $X$ any paracompact differentiable manifold. The second is proved in [3, pp. 9-10]. It is a simple consequence of a theorem concerning the differentiability of the remainder term in Taylor's formula which was proved earlier for $R$ and $R^{2}$ by H. Whitney [7]. (See also [8].)

1. Preliminaries. In the sequel $X$ and $Y$ always denote open convex subsets of $R^{n}$. We denote $n$-tuples of real numbers or real functions by a bar over a symbol. For example, we denote arbitrary points in $R^{n}$ by $\bar{r}=\left(r_{1}, r_{2}, \cdots, r_{n}\right)$ and arbitrary elements in $(C(X))^{n}$, that is continuous mappings from $X$ into $R^{n}$, by $\bar{f}=\left(f_{1}, f_{2}, \cdots, f_{n}\right)$ or $\bar{g}=\left(g_{1}, g_{2}, \cdots, g_{n}\right)$. If $\bar{f}$ is in $(C(X))^{n}$ and $\phi$ is an isomorphism from $C(X)$ to $C(Y)$, then by $\phi \bar{f}$ we mean the $n$-tuple $\left(\phi f_{1}, \phi f_{2}, \cdots, \phi f_{n}\right)$ in $(C(Y))^{n}$. The projection mappings or coordinates on $X$ are denoted by $x_{1}, x_{2}, \cdots, x_{n}$. That is $x_{i}(\bar{r})=r_{i}$ for all $\bar{r}$ in $X$. Hence $\bar{x}$ is the identity mapping on $X$ and $\bar{y}=\left(y_{1}, y_{2}, \cdots, y_{n}\right)$ is the identity mapping on $Y$. We denote constant functions by boldface. For example if $a$ is a real number, then $a_{X}(\bar{r})=a$ for all $\bar{r}$ in $X$ and $a_{Y}(\bar{s})=a$ for all $\bar{s}$ in $Y$. Observe that $1_{X}$ is the multiplicative identity in $C(X)$. The set of all real constants on $X$ is denoted by $R(X)$ and on $Y$ by $\boldsymbol{R}(Y)$.

2. Construction of the homeomorphism. Our main theorem may now be stated:

(2.1) Theorem. If $X$ and $Y$ are open convex subsets of $R^{n}$ and $\phi$ is an isomorphism from $C(X)$ onto $C(Y)$, then $\phi \bar{x}$ is a homeomorphism from $Y$ onto $X$ and $\phi f=f(\phi \bar{x})$ for all $f$ in $C(X)$. 
Our proof requires several lemmas.

(2.2) Lemma. If $\phi$ is any isomorphism from $C(X)$ onto $C(Y)$, then $\phi a_{X}=a_{Y}$ for all $a_{X}$ in $R(X)$.

Proof. For each point $\bar{r}$ in $Y$, the correspondence $a \rightarrow\left(\phi a_{X}\right)(\bar{r})$ is a homomorphism $\psi_{r}$ from $R$ into $R$. Hence $\psi_{r}$ is either the zero homomorphism or the identity mapping on $R$. But since $1_{X}$ is the multiplicative identity in $C(X)$, then $\phi 1_{X}=1_{Y}$ and, therefore, $\psi_{\bar{r}} 1=\left(\phi 1_{X}\right)(\bar{r})$ $=1$ for all $\bar{r}$. Therefore $\psi_{\bar{r}}$ must be the identity mapping on $R$ for all $\bar{r}$ in $Y$ (compare [2, p. 23]).

(2.3) Lemma. If $\phi$ is an isomorphism from $C(X)$ onto $C(Y)$ and $\bar{g}$ is in $(C(X))^{n}$, then $(\phi \bar{g})(Y)=\bar{g}(X)$.

Proof. Let $\bar{a}$ be any point of $R^{n}$ and define

$$
f_{\bar{a}}=\sum_{i=1}^{n}\left(g_{i}-\boldsymbol{a}_{i X}\right)^{2} .
$$

then $f_{\bar{a}}$ is in $C(X)$ and has a multiplicative inverse in $C(X)$ if and only if $\bar{a}$ is in $R^{n}-\bar{g}(X)$. Hence $\phi f_{\bar{a}}$ has a multiplicative inverse in $C(Y)$ if and only if $\bar{a}$ is in $R^{n}-\bar{g}(X)$. But $\phi f_{\bar{a}}=\sum_{i=1}^{n}\left(\phi g_{i}-a_{i Y}\right)^{2}$ has a multiplicative inverse in $C(Y)$ if and only if $\bar{a}$ is in $R^{n}-(\phi \bar{g})(Y)$.

(2.4) Lemma. If $\phi$ is an isomorphism from $C(X)$ onto $C(Y)$, then $\phi(f \circ \bar{g})=f(\phi \bar{g})$ for all $f$ in $C^{\infty}(X)$ and $\bar{g}$ in $(C(X))^{n}$ such that $\bar{g}$ maps $X$ into $X$.

Proof. By (0.2)

$$
f(\bar{r})=f(\bar{a})+\sum_{i}\left(r_{i}-a_{i}\right) f_{\bar{a}, i}(\bar{r})
$$

for all $\bar{a}, \bar{r}$ in $X$. Substituting $\bar{g}$ for $\bar{r}$, we obtain

$$
f \circ \bar{g}=\boldsymbol{f}(\overline{\mathbf{a}})_{X}+\sum_{i}\left(g_{i}-\boldsymbol{a}_{i X}\right) f_{\bar{a}, i} \circ \bar{g} .
$$

Hence by Lemma (2.2)

$$
(\phi(f \circ \bar{g}))(\bar{s})=f(\bar{a})+\sum_{i}\left[\left(\phi g_{i}\right)(\bar{s})-a_{i}\right] \cdot\left[\left(\phi\left(f_{\bar{a}, i} \circ \bar{g}\right)\right)(\bar{s})\right]
$$

for all $\bar{a}$ in $X$ and $\bar{s}$ in $Y$. Since $(\phi \bar{g})(\bar{s})$ is in $X$ by (2.3), then we may set $\bar{a}=(\phi \bar{g})(\bar{s})$, obtaining $(\phi(f \circ \bar{g}))(\bar{s})=f((\phi \bar{g})(\bar{s}))$ for all $\bar{s}$ in $Y$.

We now use $(0.1)$ to extend (2.4) to all functions $f$ in $C(X)$. 
(2.5) Lemma. If $\phi$ is an isomorphism from $C(X)$ onto $C(Y)$ and $\bar{g}$ in $(C(X))^{n}$ maps $X$ into $X$, then $\phi(f \circ \bar{g})=f(\phi \bar{g})$ for all $f$ in $C(X)$.

Proof. By $(0.1)$, for each $f$ in $C(X)$ and each $\epsilon>0$, there is a corresponding $f_{\epsilon}$ in $C^{\infty}(X)$ such that

$$
f_{\epsilon}(\bar{r})-\epsilon \leqq f(\bar{r}) \leqq f_{\epsilon}(\bar{r})+\epsilon \quad \text { for all } \quad \bar{r} \text { in } X .
$$

Hence

$$
\left(f_{\mathrm{c}} \circ \bar{g}\right)-\boldsymbol{\varepsilon}_{X} \leqq f \circ \bar{g} \leqq\left(f_{\mathrm{\epsilon}} \circ \bar{g}\right)+\boldsymbol{\varepsilon}_{\boldsymbol{X}} .
$$

Since a function in $C(X)$ is nonnegative on $X$ if and only if it has a square root in $C(X)$, the isomorphism $\phi$ is order-preserving. Therefore by $(2.4)$

$$
\begin{gathered}
f_{\epsilon}(\phi \bar{g})-\varepsilon_{Y}=\phi\left[\left(f_{\epsilon} \circ \bar{g}\right)-\varepsilon_{X}\right] \leqq \phi(f \circ \bar{g}) \leqq \phi\left[\left(f_{\epsilon} \circ \bar{g}\right)+\varepsilon_{X}\right] \\
=f_{\epsilon}(\phi \bar{g})+\varepsilon_{Y} \quad \text { for all } \epsilon>0 .
\end{gathered}
$$

But since $(\phi \bar{g})(Y)=\bar{g}(X) \subseteq X$, we may substitute $\phi \bar{g}$ for $\bar{r}$ in (2.5.1) to obtain

$$
f_{\epsilon}(\phi \bar{g})-\varepsilon_{Y} \leqq f(\phi \bar{g}) \leqq f_{\epsilon}(\phi \bar{g})+\varepsilon_{Y} \quad \text { for all } \epsilon>0 .
$$

Comparing (2.5.2) and (2.5.3) we have the desired result.

(2.6) Corollary. If $\phi$ is an isomorphism from $C(X)$ onto $C(Y)$, $\bar{f}$ is a continuous map from $X$ into $R^{n}$, and $\bar{g}$ is a continuous map from $X$ into $X$, then $\phi(\bar{f} \circ \bar{g})=\bar{f}(\phi \bar{g})$.

That $\phi f=f(\phi \bar{x})$ is now a special case of (2.5). To complete the proof of Theorem 2.1, we show that $\phi \bar{x}$ is a homeomorphism from $Y$ onto $X$. Clearly $\phi \bar{x}$ is a continuous map from $Y$ into $R^{n}$. By (2.3) $\phi \bar{x}$ maps $Y$ onto $X$. If we interchange $Y$ and $X$ in (2.3) and replace $\phi$ by $\phi^{-1}$, then we see that $\phi^{-1} \bar{y}$ maps $X$ onto $Y$. If we substitute $\phi^{-1} \bar{y}$ for $\bar{f}$ and $\bar{x}$ for $\bar{g}$ in (2.6), we obtain

$$
\phi\left(\left(\phi^{-1} \bar{y}\right) \circ \bar{x}\right)=\left(\phi^{-1} \bar{y}\right) \circ(\phi \bar{x}) .
$$

But since $\bar{x}$ is the identity map on $X$,

$$
\phi\left(\left(\phi^{-1} \bar{y}\right) \circ \bar{x}\right)=\phi\left(\phi^{-1} \bar{y}\right)=\bar{y} .
$$

Hence $\phi \bar{x}$ has a continuous inverse $\phi^{-1} \bar{y}$. Therefore $\phi \bar{x}$ is a homeomorphism from $Y$ onto $X$ and Theorem 2.1 is proved.

If $Y=X$ and we replace $\phi^{-1}$ in the above discussion by an arbitrary automorphism on $C(X)$, we obtain $\phi(\psi \bar{x})=(\psi \bar{x}) \circ(\phi \bar{x})$. It follows that the correspondence $\phi \rightarrow \phi \bar{x}$ is an anti-isomorphism from the group 
of all automorphisms on $C(X)$ onto the group of all homeomorphisms on $X$.

In Lemmas (2.2), (2.3), (2.4), and (2.6) we could have replaced the rings $C(X)$ and $C(Y)$ by the rings $C^{\infty}(X)$ and $C^{\infty}(Y)$, obtaining:

(2.7) Lemma. If $\phi$ is an isomorphism from $C^{\infty}(X)$ onto $C^{\infty}(Y), \bar{f}$ and $\bar{g}$ are in $\left(C^{\infty}(X)\right)^{n}$, and $\bar{g}$ maps $X$ into $X$, then

(i) $(\phi \bar{f})(Y)=\bar{f}(X)$,

(ii) $\phi(\bar{f} \circ \bar{g})=\bar{f}(\phi \bar{g})$, and for any function $f$ in $C^{\infty}(X)$

(iii) $\phi f=f(\phi \bar{x})$.

That $\phi \bar{x}$ is a diffeomorphism if $\phi$ is an isomorphism from $C^{\infty}(X)$ onto $C^{\infty}(Y)$ may be proved in the same way that we proved above that $\phi \bar{x}$ is a homeomorphism if $\phi$ is an isomorphism from $C(X)$ onto $C(Y)$. Hence:

(2.8) Theorem. If $X$ and $Y$ are open convex subsets of $R^{n}$ and $\phi$ is an isomorphism from $C^{\infty}(X)$ onto $C^{\infty}(Y)$, then $\phi \bar{x}$ is a diffeomorphism from $Y$ onto $X$ and $\phi f=f(\phi \bar{x})$ for all $f$ in $C(X)$.

\section{BIBLIOGRAPHY}

1. I. Gelfand and A. Kolmogoroff, On rings of continuous functions on topological spaces, C. R. (Doklady) Acad. Sci. URSS 22 (1939), 11-15.

2. Leonard Gillman and Meyer Jerison, Rings of continuous functions, Van Nostrand, Princeton, N. J., 1960.

3. Sigurdur Helgason, Differential geometry and symmetric spaces, Academic Press, New York, 1962.

4. Edwin Hewitt, Rings of real-valued continuous functions. I, Trans. Amer. Math. Soc. 64 (1948), 45-99.

5. Lyle E. Pursell, An algebraic characterization of fixed ideals in certain function rings, Pacific J. Math. 5 (1955), 963-969.

6. - Uniform approximation of real continuous functions on the real line by infinitely differentiable functions, Math. Mag. 40 (1967), 263-265.

7. Hassler Whitney, Differentiability of the remainder term in Taylor's formula, Duke Math. J. 10 (1943), 153-158.

8. Solution to problem E1789, Amer. Math. Monthly 73 (1966), 779-780.

Grinnell College and

UNiversity of Missouri at Rolla 